\title{
Optimization of Carotenoid Production by Paracoccus beibuensis Isolated from Lonar Crater
}

\author{
Deepti D. Dhere ${ }^{1}$, S. M. Dharmadhikari² \\ 1,2 Department of Microbiology, Government Institute of Science, Caves Road, Dr. Babasaheb Ambedkar Marathwada University, \\ Aurangabad, (M.S.), India
}

\begin{abstract}
Background: Carotenoid is terpenoid pigment having antioxidant activities and many therapeutic applications. Results: In current study, an orange pigment producing bacteria, Paracoccus beibuensis was isolated from Lonar crater in Buldhana District, Maharashtra, India. Methanolic extract of orange pigment produced from Paracoccus beibuensis was analyzed by spectrophotometer within 400-600nm and $\lambda$ max was found at 472nm with one peak spectra and shoulder region which is characteristic of Carotenoid i.e. Astaxanthin. The cultivation condition for orange pigment production were optimized by using one at a time approach as-initial pH 9.5, incubation temperature $30^{\circ} \mathrm{C}, 120 \mathrm{rpm}$ as agitation and aeration condition, $72 \mathrm{~h}$ as time of incubation, $1 \%$ inoculum,25mL volume of media in $100 \mathrm{ml}$ capacity flask and incubation in white light. Crude extraction of pigment was done and it analyzed by TLC, where Rf value of hypophase was found 0.63 which suggest the presence of xanthophyll. Under optimal conditions, the yield of orange pigment was achieved as $1 \mathrm{mg} / \mathrm{L}$. Conclusion: The extracted pigment belongs to Xanthophyll which are oxygenated carotenoid. Initial studies on purification and analysis suggest presence of Astaxanthin. Astaxanthin have strong antioxidant activity and many therapeutic applications.
\end{abstract}

Keywords: Paracoccus beibuensis, optimization, Lonar crater, orange pigment, xanthophyll

\section{Introduction}

The human life is covered with different colors which play an important role in all aspects of life including food. The freshness and safety of food depends on its color. Application of synthetic color in food preparations results into safety concern due to their harmful effects on human health. Pigments are derived from various natural sources out of which microorganisms are great source of diverse natural colors which can be used in food, as feed additives as well as for drug development [1]. On the other hand, plant pigments are less stable, and are unavailable throughout the year unlike microbial pigments [2]. Microbial pigments [3] are of great interest due to their stability and year around availability and easy cultivation methods. Microorganisms produce various pigments like Carotenoids, Melanin, Flavins, Quinones, Prodigiosin and more specially Monascins, Violacin or indigo [4]. Out of all pigments Carotenoids are very important as they are involved in reducing on set of many diseases which are produced due to the free radical damage. Astaxanthin is one of the carotenoids with commercial value as food supplement and food additives for human, animals and fish [2], Paracoccus haeundaensis an Astaxanthin producing marine bacterium has been isolated and identified [5]. In the present study orange pigment producing Paracoccus beibuensis was isolated from hypersaline-hyperalkaline environment of Lonar Crater. There are no literature studies available on optimization of orange pigment production from Paracoccus beibuensis isolated from hypersaline-hyperalkaline environment and its extraction studies.

\section{Materials and Methods}

All chemicals used were high analytical grade and solvents were Qualigen grade. Chemicals were procured from Himedia, Merk and solvents were procured from SRL.

\subsection{Microorganism and growth conditions}

The bacteria used in this study was as orange pigment producing bacterium isolated from soil collected from Lonar Crater, District- Buldhana, Maharashtra, India on Nutrient broth (Himedia) with $\mathrm{pH} 8.5$ and incubated at room temperature near window in day light for $48 \mathrm{~h}$ and identified as Paracoccus beibuensis by 16s-rRNA sequencing approach (data not included). The isolated and screened strain of Paracoccus beibuensis was stored on Nutrient Agar slant with same incubation conditions during experimental studies. For inoculum production, cultures were prepared in the Nutrient broth and incubated for 24h at room temperature with shaking condition 120rpm. Biomass was harvested and suspended in sterile physiological saline; the concentration was adjusted to 1 unit Optical Density at $660 \mathrm{~nm}$ and $1 \%$ volume of this suspension was used to inoculate the liquid medium for Carotenoid production. Cultures were prepared in Erlenmeyer flasks $(100 \mathrm{~mL})$ containing $25 \mathrm{~mL}$ of Nutrient Broth (Himedia). The initial $\mathrm{pH}$ was adjusted to 9.5 which was $\mathrm{pH}$ optima observed from qualitative analysis during screening.

2.2Standardization of physiological parameters for optimum pigment production

The effect of various culture conditions like, different incubation temperature $\left(20,25,30,35\right.$ and $\left.40^{\circ} \mathrm{C}\right)$, initial $\mathrm{pH}$ (7.5,8.0,8.5,9.0,9.5,10.0,10.5 and 11.0), incubation period (1to 5 days), inoculum \% ( 0.5 to 2.5 ), and incubation conditions like shaking (120rpm) compared with static condition, and light condition compared with incubation in dark. Culture volume $(25-100 \mathrm{~mL})$ on growth and pigment production was studied separately. The growth and pigment production determined separately in terms of wet weight of pellet $(\mathrm{g})$ and optical density of extracted pigment at its $\lambda \max$ 


\section{International Journal of Science and Research (IJSR) \\ ISSN (Online): 2319-7064 \\ Index Copernicus Value (2015): 78.96 | Impact Factor (2015): 6.391}

respectively. Degree of pigmentation which is a ratio of pigment produced to biomass produced was also analyzed.

\subsection{Standardization of medium}

Nutrient Broth, Luria Broth, Mac Conkeys Broth and Peptone water were inoculated and incubated in shaking condition at $30^{\circ} \mathrm{C}$ for $72 \mathrm{~h}$ and after incubation growth and pigment production was determined separately to know optimum medium for pigment production.

\subsection{Pigment Extraction and $\lambda \max$ determination}

The organism was inoculated (1\%) in sterile Nutrient broth having initial $\mathrm{pH} 9.5$ and incubated at $30^{\circ} \mathrm{C}$ for $72 \mathrm{~h}$ with shaking condition $(120 \mathrm{rpm})$ in white light. The extraction was done by slight modification of the procedure used by Manish R. Bhat et al; [2]. The culture medium was centrifuged (REMI-cooling ultra-centrifuge Model-C-24BL) at $8000 \mathrm{rpm}$ for $15 \mathrm{~min}$ at $4^{0} \mathrm{C}$ to separate cells. Separated cells were washed twice with sterile distilled water by centrifugation at $8000 \mathrm{rpm}$ for $10 \mathrm{~min}$. The cell pellet was suspended in Methanol and heated at $60^{\circ} \mathrm{C}$ for $20 \mathrm{~min}$ followed by centrifugation at $8000 \mathrm{rpm}$ for $10 \mathrm{~min}$ at $4^{0} \mathrm{C}$. The colored supernatant was collected and process was repeated until pellet turned white. The $\lambda$ max of supernatant was determined by scanning the crude extract for 400$600 \mathrm{~nm}$ by using spectrophotometer against methanol as reference.

\subsection{Partial pigment identification}

Identification of orange pigment was done by spectrophotometric analysis and chemical identification of methanolic extract of pigment.

\subsubsection{Spectrophotometric Analysis}

The absorption spectrum of crude pigment extract was measured within range of $400-600 \mathrm{~nm}$ using Methanol as reference [6]

\subsubsection{Chemical Identification}

For chemical identification $1 \mathrm{~g}$ dry wet of harvested cells was taken in dry tube and $10 \mathrm{~mL}$ of chloroform was added followed by vigorous shaking. The resulting mixture was filtered using Whatmann Filter paper no.1.Few drops of $85 \%$ sulfuric acid were added and observed for presence of blue colored ring [7].

\subsection{Pigment separation}

The pigment extraction was done by using procedure Mentioned by Fogg and Belcher [8]. The methanolic extract of pigment was concentrated by evaporation at room temperature. The $2 \mathrm{~mL}$ of obtained concentrate was transferred in $5 \mathrm{~mL}$ petroleum ether and partitioned with equal volume of $90 \%$ methanol followed by vigorous shaking in separating funnel. Epiphase and hypophase were used for TLC analysis.

\subsection{Analysis of pigment by TLC}

Silica gels coated on glass sheet were used as stationary phase. Few drops from each phase were spotted on baseline of TLC plate and then plate was placed inside prostrated TLC chamber containing mobile phase (methanol: benzene: ethyl acetate 5:70:25) [9]. The solvent was allowed to run till it reaches $3 / 4^{\text {th }}$ of the plate. The Chromatogram was analyzed visually for banding pattern and spot were marked. Relative $\mathrm{Rf}$ value were calculated.

\section{Results and Discussions}

\subsection{Microorganism}

The organism under study is Gram negative, non- motile coccoid rods identified as Paracoccus beibuensis by biochemical and16s rRNA sequencing approach. It produces bright orange colonies (Figure -1) with smooth surface and entire edge on Nutrient agar (Table-1) and show $\mathrm{pH}$ tolerance in alkaline range (7-11) having $6 \% \mathrm{NaCl}$. It is catalase and oxidase positive [10].

Table 1: Colony Characters of the Paracoccus beibuensis

\begin{tabular}{|c|c|c|}
\hline S. No & Colony Character & Observation \\
\hline 1 & Size & $0.1 \mathrm{~mm}$ \\
\hline 2 & Shape & Round \\
\hline 3 & Color & Bright Orange \\
\hline 4 & Margin & Entire \\
\hline 5 & Elevation & Convex \\
\hline 6 & Consistency & Mucoid \\
\hline 7 & Opacity & Opaque \\
\hline 8 & Surface & Smooth \\
\hline 9 & Grams Nature & Negative \\
\hline 10 & Morphology & Coccoid rods \\
\hline 11 & Motility & Non-motile \\
\hline
\end{tabular}

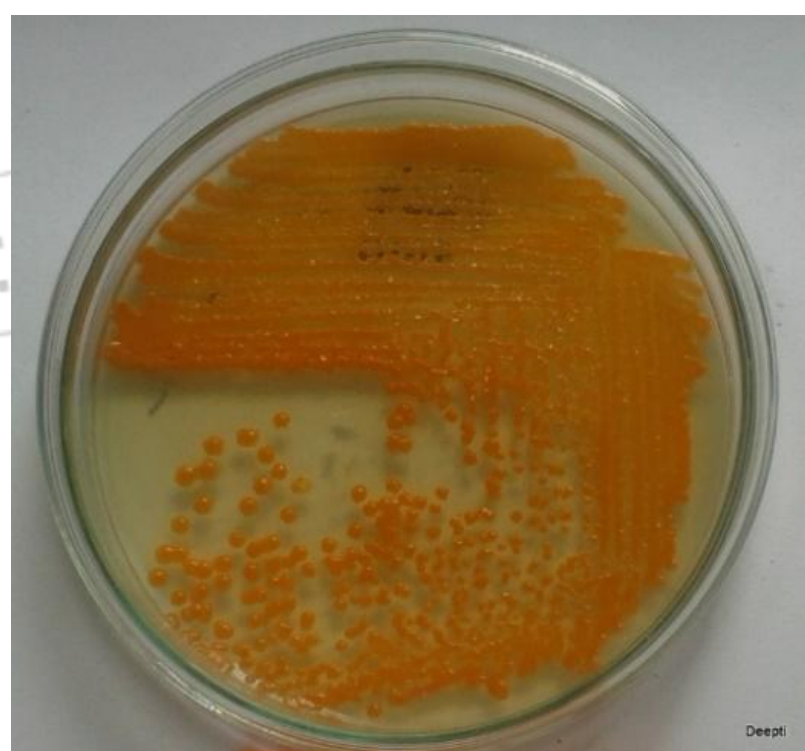

Figure 1: Isolated strain of Paracoccus beibuensis

\subsection{Partial characterization of the pigment.}

The methanolic extract of pigment was analyzed by spectrophotometer within range 400-600nm which is typical pattern of absorption spectrum of Carotenoid. Carotenoid 


\section{International Journal of Science and Research (IJSR) \\ ISSN (Online): 2319-7064}

Index Copernicus Value (2015): 78.96 | Impact Factor (2015): 6.391

absorb light in the visible region between 400-500nm [11]. The extracted pigment demonstrated the presence of shoulder region with maximum absorbance at $472 \mathrm{~nm}$ with single peak spectra which is characteristic pattern of Astaxanthin which is one of the important Carotenoids. The appearance of blue colored ring at the junction of pigment extract and sulfuric acid showed the presence of polyene pigment and it is a confirmatory test for presence of carotenoid [12] [11], similar studies on identification of chemical nature of carotenoids was done by Fatima Shatila et al; 2013 where blue colored ring was observed at the interface. Only one report on Astaxanthin producing Paracoccus beibuensis isolated from marine water, China [13] is available. There is no much literature available on extraction of pigment from Paracoccus beibuensis.

\subsection{Standardization of culture condition for optimum pigment production}

Various factors affecting on growth and pigment production by Paracoccus beibuensis were investigated. Growth and pigment production were evaluated during the investigation.

\subsubsection{Optimization of media}

Growth and pigment production were higher when the Paracoccus beibuensis was grown in Peptone water while Nutrient broth and Luria Broth (Himedia)gives same values for carotenoid produced where nutrient broth supports biomass production as compared to other tested media which was checked in terms of wet weight of pellet and negligible growth was found in the Mac Conkeys broth(Himedia) and mineral media each with $\mathrm{pH} 9.5$.

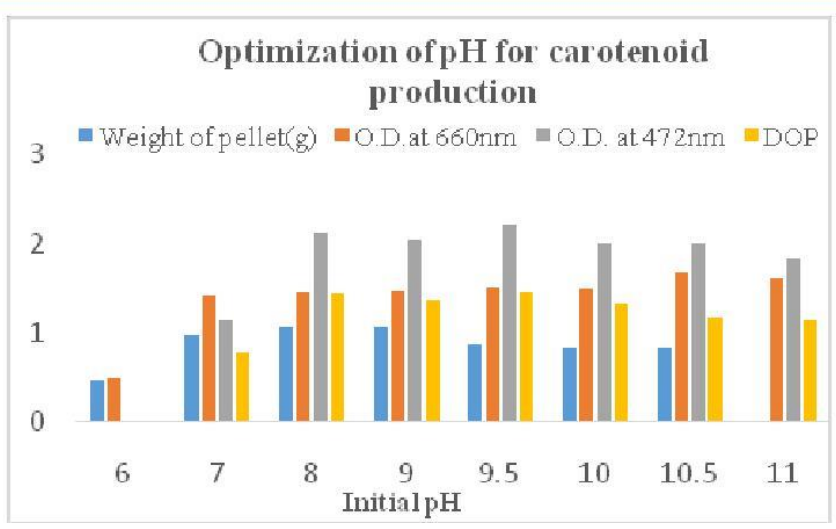

Figure 3: Optimization of initial $\mathrm{pH}$ for production of carotenoid by Paracoccus beibuensis

\subsubsection{Optimization of temperature}

Temperature is one of the most important environmental factors affecting the growth of microorganisms and it causes changes in many biosynthetic pathways, such as carotenoid biosynthesis [14]. The Paracoccus beibuensis shows highest pigment produced was at $30^{\circ} \mathrm{C}$ and it was found that as the temperature increases there is increase in biomass but pigment production was reduced (Figure 4). Similar studies on optimization was done by Bohua Wang et al; [15] and L. Hardijito et al; [16] where optimum temperature for $\beta$ carotene production was found $30^{\circ} \mathrm{C}$.

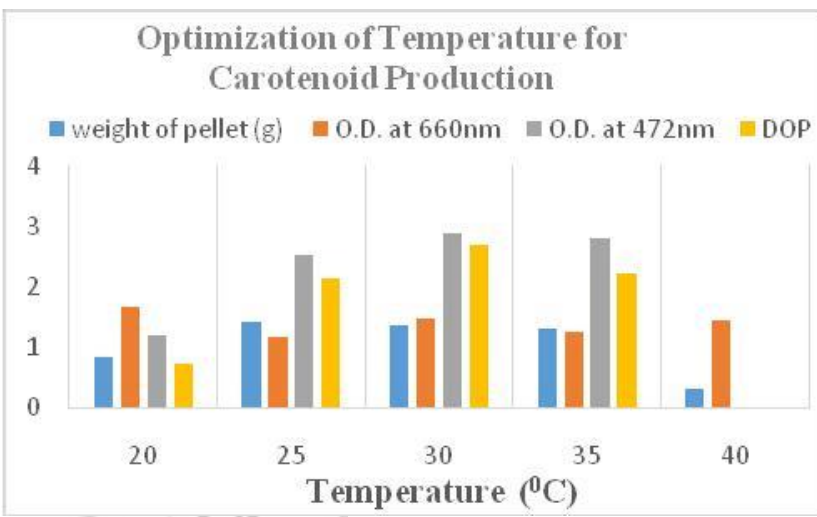

Figure 4: Optimization of Temperature for production of carotenoid from Paracoccus beibuensis

\subsubsection{Optimization of incubation conditions}

The Paracoccus beibuensis was incubated under the previously optimized condition in white light and dark separately and simultaneously. The growth and degree of pigmentation was higher in light than incubation in dark (Figure 5) which suggests that the photodynamic action on metabolism may induce the pigmentations. The light effects on metabolic activities of microorganisms and induce Carotenogenesis due to production of photo-oxidized metabolite which enhances Carotenogenic enzymes and inactivate the repressor of Carotenogenesis [17] [18]. Paracoccus beibuensis showed increased growth and degree of pigmentation production at shaking condition (120rpm) as compared to static condition (Figure -5). The data suggest that maximum growth and pigment production was obtained at shaking condition with $120 \mathrm{rpm}$. Bacteria can improve the transfer of substrate and oxygen in aerobic condition [19], similar studies were carried out by the Manish Bhat et al; [2] for production of an orange pigment where optimum shaking condition was found at $120 \mathrm{rpm}$. 


\section{International Journal of Science and Research (IJSR) \\ ISSN (Online): 2319-7064}

Index Copernicus Value (2015): 78.96 | Impact Factor (2015): 6.391

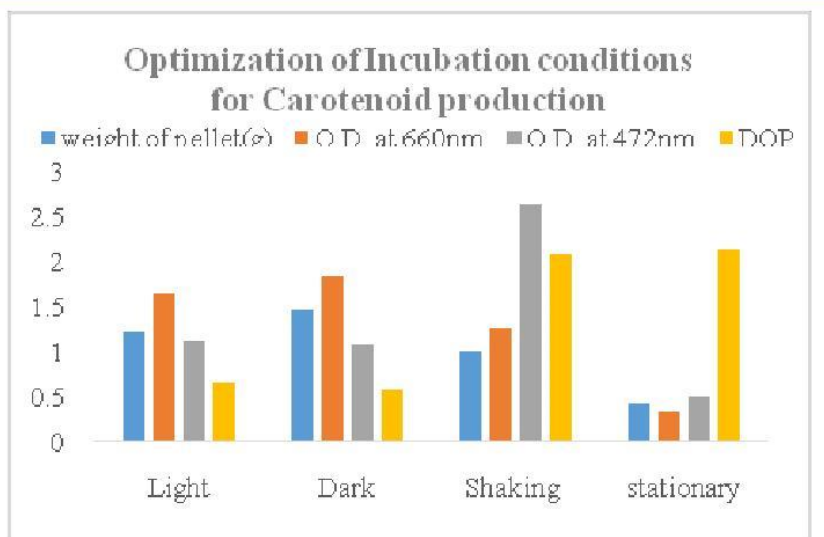

Figure 5: Optimization of Incubation conditions for production of carotenoid by Paracoccus beibuensis

\subsubsection{Optimization of volume of culture medium}

The effect of different volume of culture medium on growth and pigment production was studied in $100 \mathrm{~mL}$ Erlenmeyer flask. Paracoccus beibuensis gave maximum pigment production with $25 \mathrm{~mL}$ medium (Figure 6). The increase in the volume of the culture medium beyond the optimum volume causes decline in the growth and pigment production by strict aerobic isolates. Due to increased volume of culture medium the amount of dissolved oxygen leads to decline in growth and pigment production [20]. Manish Bhat et al; [2] optimized the media volume for carotenoid production by Salinococcus $s p$. where $100 \mathrm{~mL}$ of media gave highest carotenoid production.

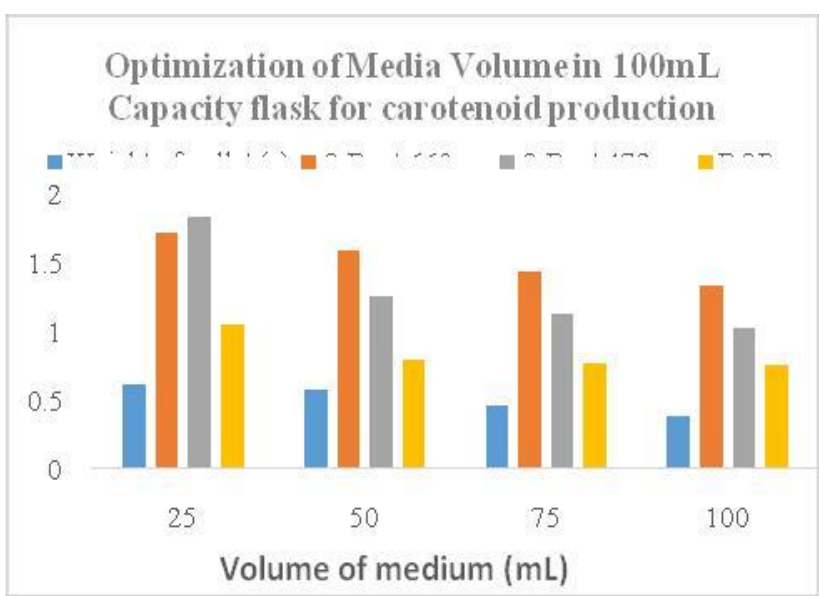

Figure 6: Optimization of media volume for production of carotenoid by Paracoccus beibuensis

\subsubsection{Optimization of incubation time}

Microorganisms generally produce the pigment up to late log phase or stationary phase. As Carotenoids are primary metabolite it shows pigment production only up to late log phase and not in stationary phase. In present study, highest pigment from Paracoccus beibuensis was observed after 3 days of incubation i.e. after $72 \mathrm{~h}$ incubation (Figure 7) [21]. Mohanty and Mukherji (2008) recorded the maximum growth and pigmentation after 48 hours. [22]

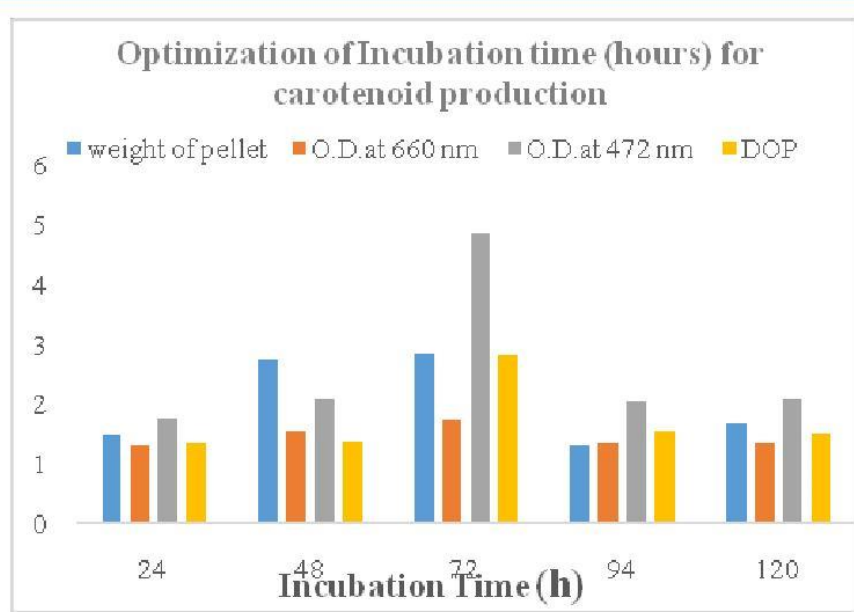

Figure 7: Optimization of incubation time for production of carotenoid from Paracoccus beibuensis

\subsection{Separation of pigment}

For separation of various components from pigment extract of Paracoccus beibuensis, $2 \mathrm{~mL}$ of concentrated pigment extract was mixed with the $5 \mathrm{~mL}$ of petroleum ether and equal volume of $90 \%$ methanol was mixed. After separation if forms two layers viz. upper petroleum ether layer called Epiphase and lower methanol layer called hypophase. The epiphasic layer i.e. upper petroleum ether layer shows the presence of yellow pigment which indicates presence of carotene. The hypophasic i.e. lower methanolic layer shows presence of Xanthophyll. As the Xanthophyll contains hydroxyl group it remains in the hypophase and $\beta$-carotene do not contain any hydroxyl group it remains in the Epiphase i.e. upper phase. The Rf value of the hypophase was 0.46 which is similar with the $\mathrm{Rf}$ of the xanthophyll i.e. Astaxanthin. Similar extraction studies were carried out by Manish Bhat et al; [2] where the absorption maxima of the pigment was found to be $450 \mathrm{~nm}$, characteristic of carotenoid pigment.

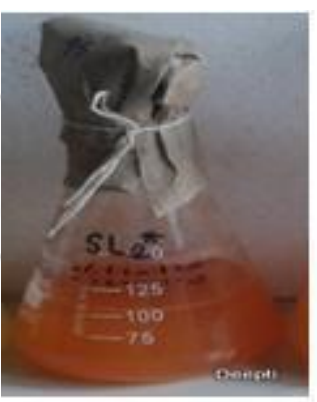

A.

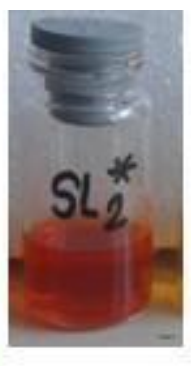

B.

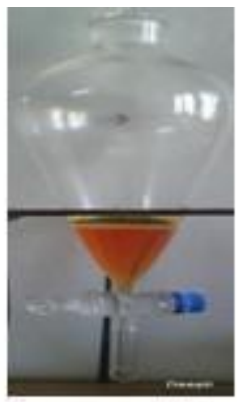

C.
Figure 8: Production and partial purification of orange pigment from Paracoccus beibuensis A- production of carotenoid from Paracoccus beibuensis, B-Extraction and partial purification of carotenoid, C-hypophase from extract

\subsection{TLC analysis of the pigment}

The epiphasic and hypophasic layers were analyzed further by Thin layer chromatography (TLC), which suggest the presence of both carotene and xanthophyll on separation i.e. Xanthophyll in the hypophase with bright orange pigment and carotene in the Epiphase with light yellow pigment. Carotenes migrates with the solvent front, while dihydroxylated compounds remain to the baseline of the 


\section{International Journal of Science and Research (IJSR) \\ ISSN (Online): 2319-7064}

Index Copernicus Value (2015): 78.96 | Impact Factor (2015): 6.391

chromatography sheet [23] (Figure 9). Hypophase with bright orange pigmented compound with value of 0.46 indicating presence of Xanthophyll i.e. Astaxanthin.

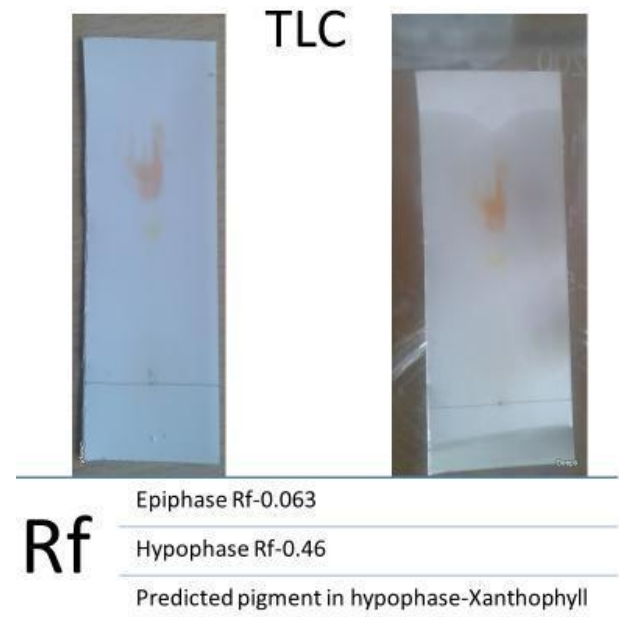

Figure 9: TLC profile of Partially purified carotenoid

\section{Acknowledgement}

Authors are thankful to Director, Government Institute of Science, Staff of Microbiology Department and Research colleagues for their moral support and constant source of inspiration

\section{References}

[1] M. H. Stafnes, Dybwad M., Brunsvik A., Bruheim P. Large scale MALDI-TOF MS based taxa identification to identify novel pigment producers in marine bacterial culture collection. Antonie Van Leeuwenhoek; 103(3):603-15, 2013

[2] R.B. Manish, Thankamani M. Media optimization. Extraction and Partial Characterization of an Orange Pigment from Salinococcus sp. MKJ997975. Int. J. of Life sci. and Pharma Research.4(2): 85-89,2015

[3] K. V. Chidambaram and L. Perumalsa, "An insightful overview on microbial pigment Prodigiosin," Electronic Journal of Biology, (5), 49-61.2009

[4] L. Dufosse, "Pigments microbial," Encyclopedia Microbiol., 4, 457-471,2009

[5] J. H. Lee, Y. S. Kim, T. J. Choi, W. J. Lee, and Y. T. Kim, "Paracoccus haeundaensis sp. Nov., a Gram negative, halophilic, Astaxanthin producing bacterium, International Journal of Systematic and Evolutionary Microbiology; 54, 1699-1702,2004

[6] A.V. Medicharla, Jagannadha Rao J and Shivaji S, The major carotenoid pigment of psychrotrophic Micrococcus roseus strain: Purification,structure and interaction with synthetic membranes,J.Bacteriol., 173, 7911-7917,1999

[7] Fatima Shatila, Hoda Yusef and Holail Hanafy, Pigment production by Exiguobacterium aurantiacum $\mathrm{FH}$, a novel Lebanese strain. International journal of Curr. Microbiol. and Appl. Sci. 2(12), pp:176-191,2013

[8] G. E. Fogg and Belcher, J.H. Pigments from Bottom deposits of an English Lake. New Phytol.60, 129142.1961
[9] E. Forgacs and T. Caserhati, "Thin-layer chromatography of natural pigments: New advances," J. Liquid Related, 25, pp. 1521-1541, 2002.

[10] D. D. Dhere, S. M. Dharmadhikari, "Bioprospecting of Haloalkalotolerent bacteria from Lonar Crater for

Production of Carotenoids", Int. J. Scientific Research and Review, 5 (3) 2016

[11]F. Shla and Y. Hyolai, "Pigment production by Exiguobacterium antc. FH, a novel Labanes strain," Int. J. Cur. Micobl. Ap. Sci., vol. 2, pp. 76-91, Dec. 2013.

[12] I. A. Ajayi, O. Ajibade, and R. A. Oderinde, "Preliminary phytochemical analysis of some plant seeds," Res. J. Chem. Sci., vol. 1, pp. 58-62, June 2011.

[13] Qiang Zheng et al; Paracoccus beibuensis sp. Nov., Isolated from the south China Sea. Curr Microbiol, 62:710-714, 2011

[14] F. Khodaiyan, S. H. Razavi, Z. E. Djomeh, and S. M. Mousavi, "Optimization of canthaxanthin production by Dietzia natronolimnaea HS-1 using response surface methodology," Pakistan. J. Biol. Sci., (10) 25442552,2007

[15] Bohua Wang et al; Optimization of $\beta$-Carotene production by newly isolated Serratia marcescens strain, Elect J. Biotechnol. (15): 1-8,2012

[16] L. Hardijito, A. Huq, and R. R. Colwell, "The Influence of environmental conditions on the production of pigment by Serratia marcescens," Bsiotechnol. Bioprocess Engineer., (7), 100-104,2002

[17] P. P. Batra, "Mechanism of photoinduced carotenoid synthesis," The J. Biol. Chem., vol. 242, pp. 5630-5635, 1967.

[18] H. Linden, P. Ballario, and G. Macino, "Blue light regulation in Neurospora crassa," Fungal Genetics and Biology, 22, pp. 141-150, 1997.

[19] E. Valduga, P. O. Tatsch, L. Tiggemann, J. Zeni, R. Colet, J. M. Cansian, et al., "Evaluation of the conditions of carotenoids production in a synthetic medium by Sporidiobolus salmonicolor (CBS 2636) in a bioreactor," Inter. J. Food Technol., 44, pp. 24452451,2009

[20] G. Goswami, S. Chaudhuri, and D. Dutta, "Effect of pH and temperature on pigment production from an isolated bacterium," Chemical Engineer. Trans., 20, pp. 127$132,2010$.

[21] B. Kaur, D. Chakraborty, and H. Kaur, "Production and evaluation of physicochemical properties of red pigment from Monascus purpureus MTCC 410," The Internet. J. Microbiol., 7, 2009.

[22] Mohanty, G., and Mukharji, S. Enhancement of NAPL bioavailablity by induction of cell -surface hydrophobicity in Exiguobacterium aurantiacum and Hurkholderia cepacia. Indian J. Biotechnol. 7:295-306, 2008.

[23] P. Mishra and N. K. Singh, "Spectrophotometric and TLC based characterization of Kernel carotenoids in short duration maize," Maydica., vol. 55, pp. 95-100, 2010 . 


\section{International Journal of Science and Research (IJSR) \\ ISSN (Online): 2319-7064}

Index Copernicus Value (2015): 78.96 | Impact Factor (2015): 6.391

\section{Author Profile}

Deepti D. Dhere received the B.Sc. degree from Dnyanopasak Mahavidyalaya, Parbhani, Maharashtra, India and M.Sc. degrees in Microbiology from Government Institute of Science, Aurangabad, M.S., India in 2006 and 2008 , respectively. After completion of postgraduation, she worked as research fellow on
Major Research Project funded by UGC Delhi, India. Now she is pursuing for her Ph.D. Degree under supervision of Dr. Mrs. S. M. Dharmadhikari at Government Institute of Science, Aurangabad, M.S., India. 